Article

\title{
Combining Planar Laser-Induced Fluorescence with Stagnation Point Flows for Small Single-Crystal Model Catalysts: CO Oxidation on a Pd(100)
}

\author{
Jianfeng Zhou ${ }^{1}{ }^{(\mathbb{D}}$, Sebastian Matera ${ }^{2}$, Sebastian Pfaff ${ }^{1}$, Sara Blomberg ${ }^{3,4}$, Edvin Lundgren ${ }^{5}$ and \\ Johan Zetterberg $1, *(1)$ \\ 1 Division of Combustion Physics, Lund University, SE-22100 Lund, Sweden; jianfeng.zhou@forbrf.lth.se (J.Z.); \\ Sebastian.pfaff@forbrf.lth.se (S.P.) \\ 2 Institute for Mathematics, Freie Universität Berlin, D-14195 Berlin, Germany; matera@math.fu-berlin.de \\ 3 Department of Chemical Engineering, Lund University, SE-22100 Lund, Sweden; \\ Sara.blomberg@chemeng.lth.se \\ 4 Advanced Light Source, Lawrence Berkeley National Laboratory, Berkeley, CA 94720-8229, USA \\ 5 Division of Synchrotron Radiation Research, Lund University, SE-22100 Lund, Sweden; \\ Edvin.lundgren@sljus.lu.se \\ * Correspondence: johan.zetterberg@forbrf.lth.se
}

Received: 10 April 2019; Accepted: 16 May 2019; Published: 25 May 2019

\begin{abstract}
A stagnation flow reactor has been designed and characterized for both experimental and modeling studies of single-crystal model catalysts in heterogeneous catalysis. Using CO oxidation over a Pd(100) single crystal as a showcase, we have employed planar laser-induced fluorescence (PLIF) to visualize the $\mathrm{CO}_{2}$ distribution over the catalyst under reaction conditions and subsequently used the 2D spatially resolved gas phase data to characterize the stagnation flow reactor. From a comparison of the experimental data and the stagnation flow model, it was found that characteristic stagnation flow can be achieved with the reactor. Furthermore, the combined stagnation flow/PLIF/modeling approach makes it possible to estimate the turnover frequency (TOF) of the catalytic surface from the measured $\mathrm{CO}_{2}$ concentration profiles above the surface and to predict the $\mathrm{CO}_{2}, \mathrm{CO}$ and $\mathrm{O}_{2}$ concentrations at the surface under reaction conditions.
\end{abstract}

Keywords: planar laser-induced fluorescence; stagnation flow; CO oxidation; Pd(100)

\section{Introduction}

Most of our atomic-scale understanding of heterogeneous catalysis stems from surface science experiments performed under well-controlled conditions, i.e., at low temperatures and at ultra-high vacuum (UHV). These conditions differ greatly from those in industrial catalysis. The awareness of the so-called "pressure gap" has in recent years resulted in an increasing number of in situ surface sensitive techniques targeting at more realistic gas conditions [1,2]. Prominent examples are near-ambient-pressure X-ray photoelectron spectroscopy (AP-XPS) [3-5], high-pressure scanning tunneling microscopy/atomic force microscopy (HPSTM/AFM) [6,7], polarization-modulated infrared absorption spectroscopy (PM-IRAS) [8,9], and surface X-ray diffraction (SXRD) [10,11].

As the pressure increases, the gas phase above a catalyst surface starts to play a complex role [12]. In semi-realistic conditions, the gas phase transport is not ballistic anymore and a product boundary layer (BL) might form above the catalyst, hindering the reactants from efficiently reaching the catalytic surface. As a consequence, the gas concentrations at the catalyst surface might differ significantly from the ones applied at the inlet, depending on the activity of the catalyst [13-15]. This coupling of surface chemistry and gas transport not only has a quantitative effect, but qualitatively new features 
might arise, such as hysteresis or strong lateral variation of the reaction conditions and concomitant reactivity and surface atomic structure $[15,16]$. Experimentally, the latter has been observed for the $\mathrm{CO}$ oxidation on a $\mathrm{Pd}(100)$ surface, where it has been shown that a gradient of the gas concentrations across the surface can lead to a gradient of the surface optical reflectivity, a strong indication of spatial inhomogeneity of oxide thickness or roughness on the surface $[17,18]$. For a better understanding of the surface chemistry or the catalytic processes at realistic gas conditions, knowledge of the effect of flow and reactor geometries on model catalysts becomes important $[16,19]$.

Conventional methods to acquire gas phase data include mass spectrometry (MS) and Fourier transform infrared spectrometry (FTIR). However, neither can spatially probe the gas phase close to the sample in an easy manner. With a scanning mass spectrometer, one can spatially sample the gas phase above the sample with a capillary [20]. This would, however, inherently affect the gas flow and temperature above the sample. Conventional FTIR can be modified to spatially measure the gas phase above a catalyst [21]. It is, however, a line-of-sight technique, and thus not able to resolve the gas phase in all dimensions. In this context, planar laser-induced fluorescence (PLIF) is a valuable technique that can provide 2D spatially resolved gas phase information, non-intrusively and with a spatial resolution down to $70 \mu \mathrm{m}$ [22-24].

Ideally, gas phase measurements are complemented with a theoretical/computational analysis of the coupling of gas flow and catalytic activity, e.g., by the recently developed combined first-principles kinetic Monte Carlo (1p-kmc) and computational fluid dynamics (CFD) approach [25]. With this combination, the maximum insight into the surface chemistry can be gained [26].

In general geometries, CFD might become prohibitively expensive, especially when targeting for the elucidation of the pressure/reactivity correlation, which would require solving the inverse problem. For the use of single-crystal model catalysts, stagnation flow reactors are especially appealing, as they ensure a highly homogeneous gas phase distribution across the catalyst. If properly designed, the transport can be modeled by a simple one-dimensional boundary value problem, allowing for an efficient numerical treatment $[27,28]$.

In this work, we present our attempts to realize combined PLIF/stagnation flow experiments for gas phase studies of single-crystal model catalysts and demonstrate their capabilities on the prototypical $\mathrm{CO}$ oxidation over a $\mathrm{Pd}(100)$ surface. We have used PLIF to spatially resolve the $\mathrm{CO}_{2}$ concentration distribution above the catalyst under reaction conditions. With a stagnation flow model, we extract the turnover of frequency (TOF), i.e., the rate of $\mathrm{CO}$ conversion per unit surface area, of the catalyst surface from the measured concentration of $\mathrm{CO}_{2}$ above the surface. We then use this value of the TOF to predict the concentrations of $\mathrm{CO}_{2}, \mathrm{CO}$, and $\mathrm{O}_{2}$ at the catalyst surface.

\section{Experimental Study}

\subsection{Reactor Chamber}

A schematic of our setup is shown in Figure 1. A cubical chamber of $23 \mathrm{~mL}$ volume with three windows for optical access was used for the PLIF measurements. The sample used for this study is a hat-shaped Pd single crystal with a diameter of $6 \mathrm{~mm}$, a height of $2 \mathrm{~mm}$, and with the (100) surface orientation. A pipe is positioned above the sample from the top of the chamber and used as the gas inlet, which allows for a gas stream towards the sample surface, as shown in Figure 1b. The distance between the pipe and the sample surface is $\sim 5.5 \mathrm{~mm}$. The sample was supported and heated by a boralectric heater and, the temperature was measured by a type-D thermocouple.

The inlet gas flow of each species was set by individual mass flow controllers (Bronkhorst EL-FLOW, Bronkhorst High-Tech B.V., Ruurlo, The Netherlands), and the gas pressure in the reactor was controlled by a digital pressure controller (Bronkhorst EL-PRESS) at the gas outlet. In addition to PLIF, the gas composition in the reactor was measured by a quadruple mass spectrometer (MS, Pfeiffer, QME 220, Pfeiffer Vacuum GmbH, Asslar, Germany) connected to the outlet of the reactor by a gas tube (length $=80 \mathrm{~cm}$ and diameter $=1 / 16 \mathrm{in}$ ). An automatic leak valve was used to regulate the amount of 
gas from the outlet leaking into the MS for analysis, and to stabilize the pressure in the MS at $5 \times 10^{-6}$ mbar. A more complete description of the gas system can be found in [29]. The MS signal is converted into mbar by normalizing the MS CO signal to the known initial partial pressure, and the $\mathrm{MS} \mathrm{CO}_{2}$ signal was scaled to match the measured conversion of CO.

In a stagnation flow, the gas transport in the central region above the catalyst can be modeled using the stagnation flow equations, when the diameters of the catalyst surface and the inlet are large compared to the distance between the inlet and the catalyst [27]. In this work, the diameters of the single crystal sample and the inlet are $6 \mathrm{~mm}$ and $7 \mathrm{~mm}$, respectively, which are similar to the distance between the inlet and catalyst. The deviation from the ideal case has been considered in our modeling, and we found that the necessary modifications to the model for the ideal case are rather small, such that the computational efficiency is not affected and the parameters needed for the modified model can efficiently be determined from the measured PLIF signal.
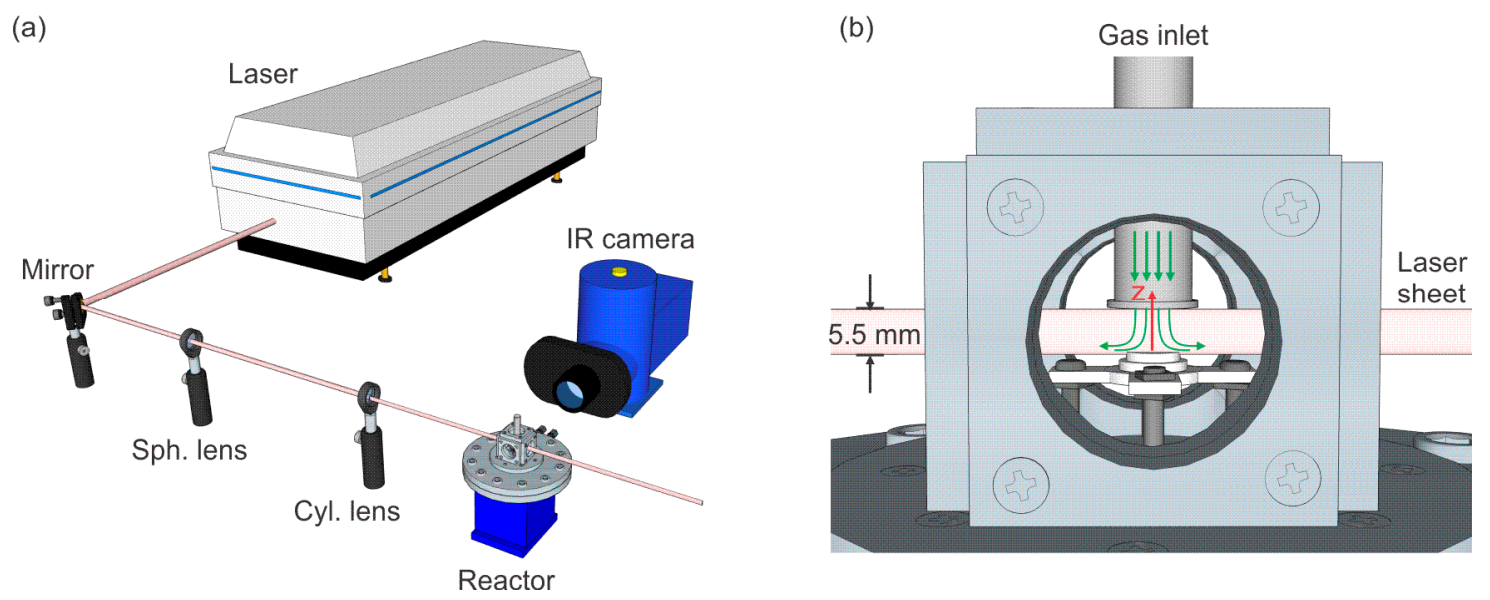

Figure 1. Schematic of the experimental setup: (a) overview and (b) a close-up of the reactor chamber with the laser sheet. The green arrows show the flow schematically, and the red arrow indicates the $\mathrm{z}$-axis in the modeling.

\subsection{PLIF}

Laser-induced fluorescence (LIF) is a highly sensitive, species-specific technique that can be used for gas detection with high spatial resolution [30-32]. As illustrated in Figure 1, when a laser beam is formed into a laser sheet using a pair of lenses, the gas molecules in a 2D region can be excited by the laser light tuned to a specific wavelength. Upon relaxation, these gas molecules fluoresce, and a camera positioned perpendicular to the laser sheet can be used to record or image the fluorescence signal (usually) at another wavelength. In this way, 2D information (e.g., concentrations and temperature) on the gas of interest can be obtained. This 2D spatially resolved technique is called planar laser-induced fluorescence (PLIF).

The experimental setup and detection scheme for the $\mathrm{CO}_{2}$ PLIF have been described in detail previously [33], so only a brief description will be given here. $\mathrm{CO}_{2}$ was excited through the $\left(00^{0} 0\right)$ $\rightarrow\left(10^{0} 1\right)$ combination band at $\sim 2.7 \mu \mathrm{m}$ and the following fluorescence from the fundamental band was detected at $\sim 4.3 \mu \mathrm{m}$. The fundamental $1064 \mathrm{~nm}$ laser beam from a single-mode Nd:YAG laser was used to pump a broadband infrared optical parametric oscillator (IR-OPO, GWU, versaScan-L 1064, Erftstadt, Germany), generating a signal beam at $\sim 1.7 \mu \mathrm{m}$ and an idler beam at $\sim 2.7 \mu \mathrm{m}$, with $\sim 8 \mathrm{~mJ} /$ pulse and $\sim 7 \mathrm{~mJ} /$ pulse, respectively, both operating at $10 \mathrm{~Hz}$. As shown in Figure 1, the idler beam was formed into a thin laser sheet of $\sim 6 \mathrm{~mm}$ height by a spherical $(\mathrm{f}=+500 \mathrm{~mm})$ and a cylindrical lens $(\mathrm{f}=+100 \mathrm{~mm})$, and then sent through the reactor between the gas inlet and the catalyst. The $\mathrm{CO}_{2}$ fluorescence was imaged by a liquid-nitrogen-cooled IR camera (Santa Barbara Focal Plane, SBF LP134, Goleta, CA, USA). An interference filter inside the camera was used to suppress the strong thermal 
background. The PLIF signal can be reliably estimated from $\sim 300 \mu \mathrm{m}$ above the sample surface, limited by the alignment of the sample with respect to the camera and the imaging system of the current setup, which gives a $\sim 70 \mu \mathrm{m} /$ pixel resolution. Quantification of the detected PLIF signal was achieved by calibration measurements with known $\mathrm{CO}_{2}$ partial pressures at similar conditions (temperatures and pressures) to real measurements. Due to the lack of a reference cell in the current setup, which can be used to compensate for wavelength drifting and energy fluctuation of the laser [23], the calibration of the $\mathrm{CO}_{2}$ signal has an estimated uncertainty of $\sim 20 \%$.

\section{3. $\mathrm{CO}_{2}$ Visualization}

For the measurements, the $\operatorname{Pd}(100)$ sample was first cleaned in a UHV chamber with several sputtering and annealing cycles, and then transferred, through open air, to the stagnation flow reactor for measurements. To reduce surface contamination from transferring the sample, the temperature of the sample was ramped up and down under $\mathrm{CO}$ oxidation reaction conditions with an excess of $\mathrm{O}_{2}$, prior to the start of the measurements. The sample temperature was monitored using a type $\mathrm{D}$ thermocouple attached to the edge of the heater, which has been calibrated using thermographic phosphors to correspond to the actual sample surface temperature.

As an example, we considered an inlet gas mixture with $92 \% \mathrm{Ar}, 4 \% \mathrm{CO}$, and $4 \% \mathrm{O}_{2}$ at a total pressure of $300 \mathrm{mbar}$ and a total flow of $200 \mathrm{~mL} / \mathrm{min}$. We ramped the temperature up and down between room temperature and $360^{\circ} \mathrm{C}$ at a rate of $10.5^{\circ} \mathrm{C} / \mathrm{min}$, which ensured quasi-stationary flows. The PLIF images in Figure 2a-c are taken during the increase of the temperature, and reveal that the sample activity has gone through three phases: i) the whole sample is in the low activity regime; ii) the sides (here referred to the rest of the sample) of the sample are highly active (indicated by the red arrow in Figure 2b), while the (100) surface is still in the low activity regime; iii) the whole sample is highly active. During the temperature ramp-down, the sample has gone through the three phases in the opposite direction, as shown by the PLIF images in Figure $2 \mathrm{~d}-\mathrm{f}$. See also Movies M1 and M2 showing the $\mathrm{CO}_{2}$ PLIF measurements during the up and down temperature ramps, respectively. By integrating the region $\sim 0.3 \mathrm{~mm}$ above the catalyst surface (the dashed line in Figure 2a), we obtain the $\mathrm{CO}_{2}$ LIF signal trends for both the up and down branches, as shown in Figure $2 \mathrm{~g}$. It is worth noting that the $\mathrm{CO}_{2}$ intensity reaches a plateau at phase iii and does not increase further with increasing temperature, indicating a mass transfer limited (MTL) reaction at the surface. By comparing the LIF signal in Figure $2 \mathrm{~g}$ with the MS signal in Figure $2 \mathrm{~h}$, the $\mathrm{CO}_{2}$ pressure close to the catalyst surface measured by PLIF is significantly higher than that measured by the MS, by a factor of 2 . In addition, there are no obvious steps in the LIF trends, while there are distinct steps in the MS trends, arising from the ignition of the sides of the sample. This demonstrates that PLIF can measure a well-defined $\mathrm{CO}_{2}$ signal generated from the (100) surface, which is not or only marginally affected by the signal generated from the sides of the sample. This is due to the feature of the stagnation flow, where the gas is pushed away from the central region above the catalyst, such that activity originating from the downstream part of the sample does not influence the gas composition over the center of the investigated catalyst surface. It is also interesting to see that the first step in the MS trend, corresponding to the activity of the sides, is much larger than the second step originating from the (100) surface. This could be due to the flow geometry. However, the strong signal generated from the sides has very little impact on the PLIF signal from the center of the sample. Thanks to this characteristic, the PLIF signal is well suited to characterize the reactor for a comparison with the stagnation flow model, as will be presented below.

The case with a non-stagnation flow geometry can be found in the supplementary information, which shows that the active sides of a $\mathrm{Pd}(111)$ sample can contribute significantly to the $\mathrm{CO}_{2}$ signal in the vicinity of the (111) surface. This has practical implications as single crystals are being widely used in in situ or operando catalysis studies, and they are in practice manufactured to have grooves or to be hat-shaped for easy-to-hold purposes. This could introduce undefined catalytic surfaces, which are often exposed to the same gas environment as the well-defined catalytic surface that is under investigation. If both are equally active, as in the cases shown above, it would be difficult 
to avoid the resulting interference using prototypical flow reactor geometries, leading to a poorly defined correlation in structure-function analysis. To overcome this problem, the presented combined stagnation flow with PLIF approach could serve as a useful alternative.
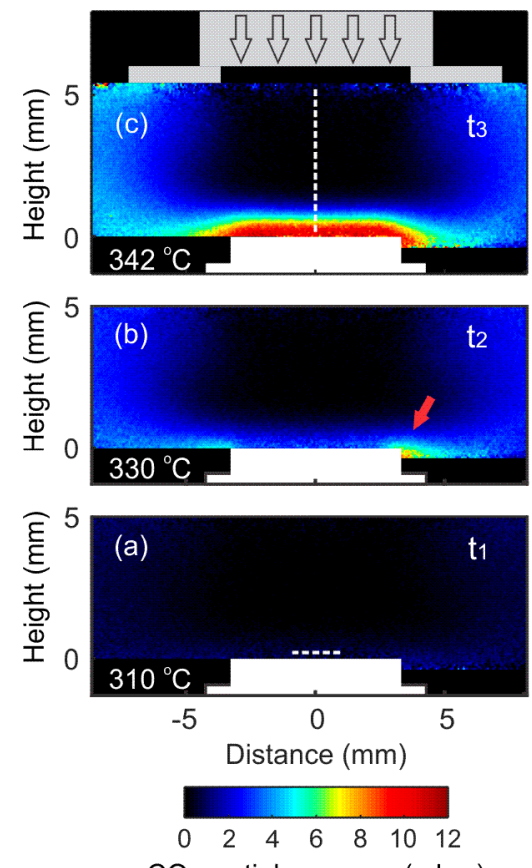

$\mathrm{CO}_{2}$ partial pressure (mbar)
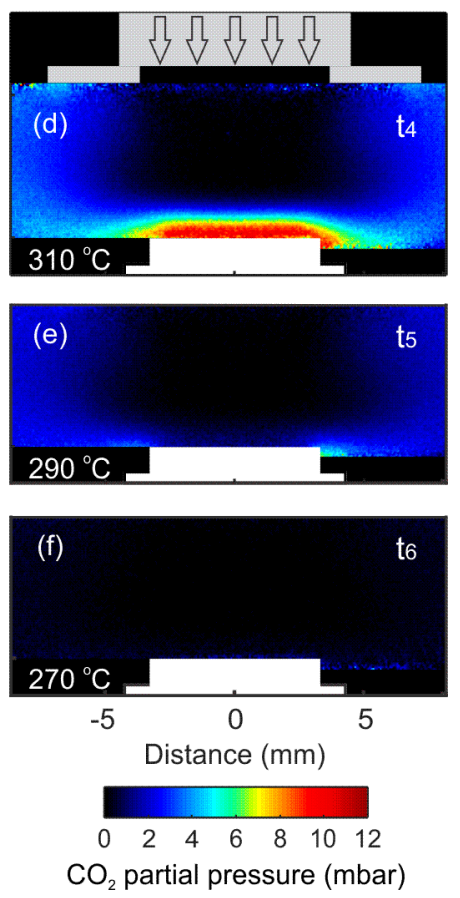
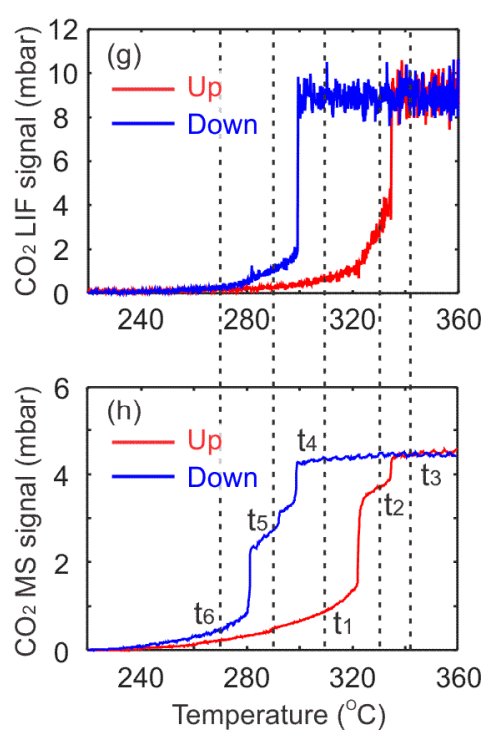

Figure 2. The $\mathrm{CO}_{2}$ distribution over a $\mathrm{Pd}(100)$ single crystal during $\mathrm{CO}$ oxidation at $200 \mathrm{~mL} / \mathrm{min}$ total flow and 300 mbar total pressure, and with 4:4:92 $\mathrm{O}_{2}: \mathrm{CO}:$ Ar ratio. (a-c), 10-shot average PLIF images recorded at different times and temperatures during the temperature ramp-up. (d-f), 10-shot average PLIF images recorded at different times and temperatures during the temperature ramp-down. (g) The integrated PLIF signal $0.3 \mathrm{~mm}$ above the sample, as indicated by the dashed line in (a). (h) The corresponding MS signal. The dashed line in (c) indicates the central region between the inlet and the surface. The red arrow in (b) indicates the active side of the sample.

\section{Modeling}

As indicated by the white dashed line in Figure 2c, the gas concentration in the central region between the catalyst and the depends only on the distance $z$ from the catalyst, as indicated by the red arrow in Figure 1b. Then the mass fractions $Y_{i}$ of the different gaseous species between the center of the catalyst $(\mathrm{z}=0)$ and the inlet $(\mathrm{z}=\mathrm{L}=5.5 \mathrm{~mm})$ can be modeled with the one-dimensional boundary value problem:

$$
\rho v_{z} \frac{\partial}{\partial z} Y_{i}-\sum_{j=1}^{N_{\text {spec. }}} \frac{\partial}{\partial z} D_{i j} \frac{\partial}{\partial z} Y_{j}=0,
$$

with (boundary conditions at inlet)

$$
Y_{i}(z=L)=Y_{i n l, i},
$$

and (boundary conditions at catalyst)

$$
-\sum_{j=1}^{N_{s p e c .}} D_{i j} \frac{\partial}{\partial z} Y_{j}(z=0)=v_{i} m_{i} T O F
$$

where the indices $i, j$ differentiate the species, $\rho$ is the mass density and $v_{z}$ is the axial velocity, both depending on the normal distance $z$ to the catalyst, $v_{i}$ is the stoichiometric coefficient, $m_{i}$ is the molecular mass, and TOF is the turn over frequency in units of reactions per unit time and unit area. For Equation (1), we assume stationary operation and negligible gas phase chemical reactions. The 
inlet mass fractions $Y_{i n l, . i}$ are given from the applied gas phase composition. The second boundary condition at $z=0$ (the position of the catalyst) accounts for the chemical conversion due to the catalyst. The diffusion coefficients $D_{i j}$ and other transports as well as thermochemical properties of the gas phase are taken to obey the commonly employed semi-empirical correlations [27]. As we are operating at a large excess of Ar and rather low velocities, the density $\rho$, the temperature $T$, the axial velocity $v_{z}$ and the diffusivities $D_{i j}$ can be assumed to be independent of the actual reactivity. Equation (1) is then linear in the mass fractions and the normalized partial pressures (NPP)

$$
p_{n, i}(z)=\frac{p_{i}(z)-p_{i n l, i}}{\int_{z_{0}}^{z_{e}} p_{i}\left(z^{\prime}\right)-p_{\text {inl },, i} d z^{\prime}},
$$

are independent of the employed catalyst and depend only on the characteristics of the reaction chamber and the flow conditions. Because of this property, these quantities are especially suited to test our model for the mass transport. The integration can be done between any two points along the z-axis for which the integral does not equal zero.

The solutions to Equations (1)-(3) require the velocity and temperature fields as an input. In principle, these could be obtained from a non-reactive CFD for the whole chamber [34]. However, besides being computationally demanding, this would require boundary conditions for the temperature at the reactor walls, to which we have no access. Instead, we will infer the relevant information from the measured NPP of $\mathrm{CO}_{2}$. We constrain $v_{z}(z)$ and $T(z)$ to obey the ideal stagnation flow equations, but with the boundary conditions at the inlet for these fields as adjustable parameter. The reason for this choice is as follows: Only within a rather thin BL above the catalyst, we have a deviation from the applied concentrations. As the BL is small compared to the catalyst diameter, the ideal stagnation flow equations will be a good approximation there. By adjusting the boundary conditions for the velocity and temperature field, we can now realize any solution to the stagnation flow equation within the BL. Outside the BL, the stagnation flow equations are not valid and therefore the velocity and temperature fields might be wrong. However, as the concentration gradients vanish there, this does not matter as Equation (1) is automatically fulfilled. In practice, we observed that variation of the inlet temperature has only a minor effect on the shape of the NPP and that we can reproduce the experimental signal sufficiently accurately by leaving the inlet radial velocity at its nominal value of zero and only increasing the axial velocity by a factor of 2 from its nominal value, defined by the applied flow rate of $200 \mathrm{~mL} / \mathrm{min}$.

The axial inlet velocity, as an input for the modeling, is determined by solving the reactive stagnation flow equations, i.e., Equations (1)-(3) together with corresponding equations for the velocity and the temperature, at a constant low TOF and adjusting its value to reproduce the experimental NPP. Our approach to solve the stagnation flow equations and the employed thermochemical and transport models can be found in [19]. Due to the linearity of Equation (1), the actual TOF responsible for the experimental signal can conveniently be determined from the TOF used for the simulation and the ratio of the denominators in the experimental and simulated NPPs. A more detailed description of the modeling can be found in the supplementary materials.

\section{Results and Discussion}

Figure 3 compares the experimental $\mathrm{CO}_{2} \mathrm{NPPs}$ (crosses) and the simulated $\mathrm{CO}_{2} \mathrm{NPPs}$ (solid lines) in the central region along the $\mathrm{z}$-axis between the inlet and the sample surface, for a range of catalyst temperatures from the up and down branches, shown in Figure 2g. The nominal inlet velocity has been increased by a factor of 1.9 and 1.8 for the up and down branches, respectively. Although the stagnation flow equation is expected to be valid in the BL, the inlet velocity parameters in principle need to be adjusted for every single case, as the flow field above the BL might depend on uncontrolled aspects of the experiment such as the temperature distribution of the reactor walls. For simplicity's sake, we have used only the two different values for the inlet velocity for the up and down branches and their values 
have been chosen to give the best visual agreement between the simulated and experimental NPPs. The larger values for the effective inlet velocity can be rationalized as follows: In contrast to the ideal case with a quasi-infinite surface, the gas can flow around the catalyst before reaching the surface and thus the boundary layer must be thinner. This corresponds to higher inlet velocities, which compress the boundary layer.

(a)

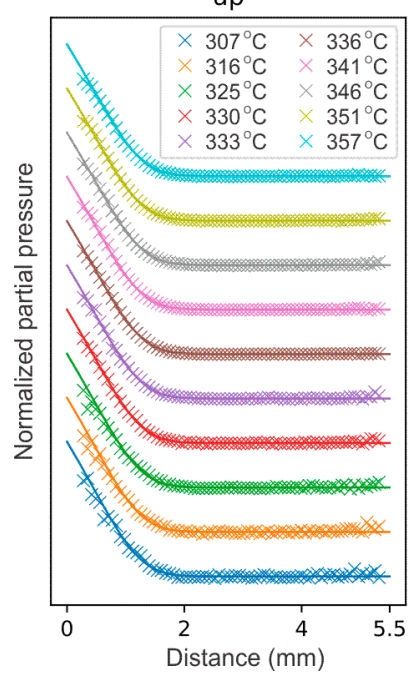

(b)

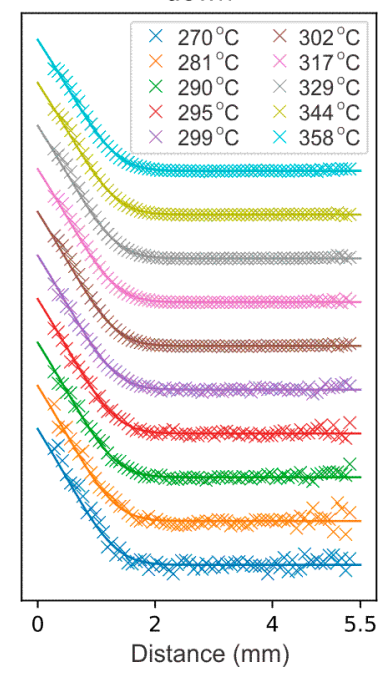

Figure 3. Comparison between the experimental $\mathrm{CO}_{2} \mathrm{NPPs}$ (crosses) and the simulated $\mathrm{CO}_{2} \mathrm{NPPs}$ (solid lines) for a range of catalyst temperatures from the temperature ramp-up (a) and -down branches (b) in Figure 2. Note that the first data point (cross) corresponds to a measured signal at $0.3 \mathrm{~mm}$ above the surface.

Our theoretical model matches the experimental findings very well, indicating that we can achieve a good stagnation flow in the reactor. As can be seen in Figure 3, the curves in both branches show a small systematic disagreement at lower temperatures. This is because the sample is not highly active at lower temperatures, thus producing only a small amount of $\mathrm{CO}_{2}$ (less than $0.5 \mathrm{mbar}$, compared to $\sim 9$ mbar when the sample is highly active), which results in a noisier PLIF signal.

Our model can now be used to extract the TOF from the experimental PLIF data from the ratio of the denominators in the experimental and simulated NPPs, following Equation (4). Figure 4 shows the TOF as a function of temperature, which is in agreement with the literature in the field. This includes features such as the hysteresis effect, which also has been previously observed [35]. The TOF is normalized, i.e., number of reactions per second and surface unit cell of $\operatorname{Pd}(100)$. The TOF estimation can then be used to predict the gas concentrations at the surface. Figure 5 shows the partial pressures of $\mathrm{CO}_{2}, \mathrm{CO}$, and $\mathrm{O}_{2}$ at the surface as a function of temperature for the up and down branches. As can be seen, the $\mathrm{CO}$ and $\mathrm{O}_{2}$ partial pressures decrease as the $\mathrm{CO}_{2}$ pressure increases. As one might expect, the relative drop in the $\mathrm{O}_{2}$ pressure is roughly half that of $\mathrm{CO}$, as we have an over-stoichiometric mixture with twice as much oxygen as needed. Notably, the $\mathrm{CO}$ partial pressure drops to a value of $\sim 3$ mbar ( $25 \%$ of its nominal value). Once this value is reached, a further increase in the temperature does not lead to lower values, indicating an MTL reaction at the surface. In the MTL regime, the $\mathrm{CO}_{2}$ pressure is modeled to be $\sim 10.5 \mathrm{mbar}$ at the surface, as shown in Figure 5a, compared to $9 \mathrm{mbar}$, as measured by PLIF at $0.3 \mathrm{~mm}$ above the surface, as shown in Figure 2g. 


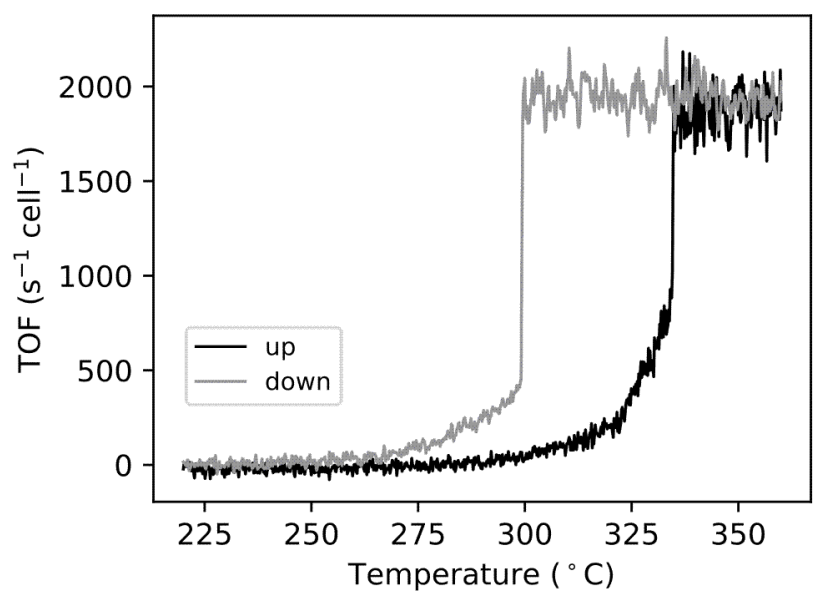

Figure 4. TOF as a function of temperature in the up and down branches.
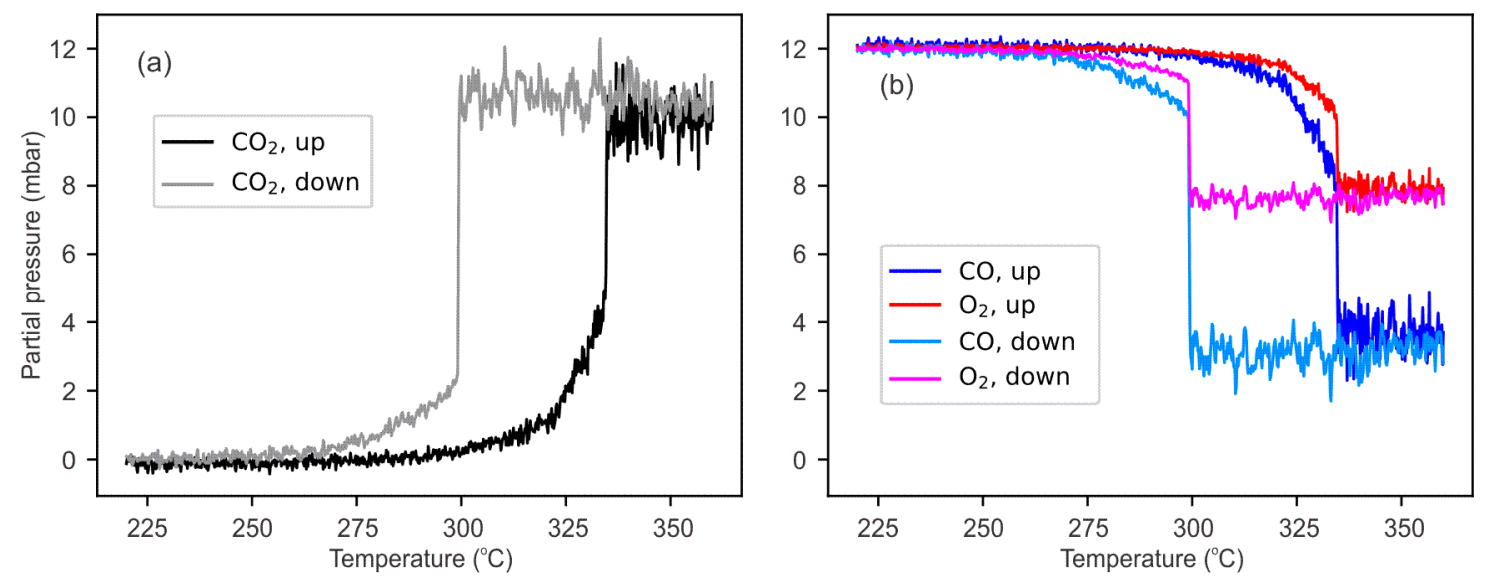

Figure 5. Simulated partial pressures at the sample surface as a function of temperature in the up and down branches. (a) $\mathrm{CO}_{2}$ profile and (b) corresponding $\mathrm{CO}$ and $\mathrm{O}_{2}$ profiles, simulated from the measured $\mathrm{CO}_{2}$ pressure profiles above the surface.

The results in this and the previous sections demonstrate the benefits of the employed stagnation flow reactor geometry combined with PLIF measurements. The flow geometry ensures a highly uniform gas phase across the catalyst surface and PLIF allows us to spatially probe the gas phase close to the surface. The combined features of stagnation flow and PLIF make it possible to measure the signal, which is only from the activity of the surface under study and does not contain any contaminations from the activity of other parts of the catalyst—as is, for instance, the case for an MS signal measured at the outlet of the reactor. Moreover, these features allow us to use a simple one-dimensional model, and the spatial resolution of the PLIF measurements allows us to adjust the flow parameters of the model. With this model, we can extract the TOF and thereby partial pressures at the surface without the need for a kinetic model of the catalyst or a full CFD model of the reactor. The approach also gives us the possibility to use smaller samples, which is more economical, especially for single-crystal measurements. It should be mentioned that this kinetic model-free analysis has some limitations. First, gas phase reactions need to be negligible, which, however, is the case for many reactions studied in surface science catalysis. Second, if there is more than one TOF, e.g., in competitive oxidation, we can only extract either only one, or a superposition of the TOFs from the PLIF signal of a single species. In these cases, we could repeat the experiment with PLIF probing of another molecule, or ideally, modify the PLIF experiment such that multiple species could be detected simultaneously [36]. Using a model for the surface kinetics could solve this problem because it would likely introduce a correlation between the different TOFs. We want to clarify that the method employed to estimate the TOF and 
the velocity parameters was more to demonstrate the suitability of the model and the relation of the parameters to the properties of the experimental signal. For obtaining more accurate parameter values, one would employ a simultaneous least square fit of all parameters. Lifting the linearity assumption would allow us to obtain results in regimes, where we cannot assume the large excess of one species.

\section{Summary and Outlook}

We have presented the gas phase study of a $\mathrm{Pd}(100)$ single crystal during $\mathrm{CO}$ oxidation to demonstrate the advantages of the combined approach of stagnation flow and PLIF, such as a highly uniform gas phase composition across the sample and measurements that are not affected by interference from the sides of the catalyst. Albeit not an ideal stagnation flow, the reactor can be analyzed using a similar simple model. With the model, we can estimate the TOF of the catalyst surface and simulate the gas concentration profiles at the surface, even without a kinetic model for the catalyst. This could only be achieved due to the combination of a dedicated reactor geometry, PLIF, and modeling that can exploit the spatially resolved gas phase information provided by PLIF.

Supplementary Materials: The following are available online at http://www.mdpi.com/2073-4344/9/5/484/s1. CO distribution over a Pd(111) single crystal in a non-stagnation flow (PDF); Reactive flow modeling (PDF); Movie M1 showing the $\mathrm{CO}_{2}$ PLIF signal in a stagnation flow during temperature ramp-up (AVI); Movie M2 showing the $\mathrm{CO}_{2}$ PLIF signal in a stagnation flow during temperature ramp-down (AVI)

Author Contributions: Conceptualization, J.Z. (Jianfeng Zhou) and J.Z.; experiment, J.Z. (Jianfeng Zhou), S.P. and S.B.; modeling, S.M.; writing - original draft preparation, J.Z. (Jianfeng Zhou) and S.M.; writing-review and editing, S.P., S.B. and E.L.; supervision, E.L. and J.Z..; funding acquisition, S.M. and J.Z.

Funding: This research was financially supported by the Knut \& Alice Wallenberg Foundation, the Swedish Research Council, the Swedish Foundation for Strategic Research, and the Einstein Foundation Berlin through the framework MATHEON/ECMath.

Conflicts of Interest: The authors declare no conflict of interest.

\section{References}

1. Rupprechter, G.; Weilach, C. Mind the gap! Spectroscopy of catalytically active phases. Nano Today 2007, 2, 20-29. [CrossRef]

2. Frenken, J.; Groot, I. (Eds.) Operando Research in Heterogeneous Catalysis; Springer: Cham, Switzerland, 2017.

3. Blomberg, S.; Hoffmann, M.J.; Gustafson, J.; Martin, N.M.; Fernandes, V.R.; Borg, A.; Liu, Z.; Chang, R.; Matera, S.; Reuter, K.; et al. In-situ X-ray photoelectron spectroscopy of model catalysts: At the edge of the gap. Phys. Rev. Lett. 2013, 110, 117601. [CrossRef]

4. Karshığlu, O.; Bluhm, H. Ambient-pressure X-ray photoelectron spectroscopy (APXPS). In Operando Research in Heterogeneous Catalysis; Frenken, J., Groot, I., Eds.; Springer International Publishing: Cham, Switzerland, 2017; pp. 31-57.

5. Toyoshima, R.; Yoshida, M.; Monya, Y.; Suzuki, K.; Mun, B.S.; Amemiya, K.; Mase, K.; Kondoh, H. Active surface oxygen for catalytic $\mathrm{CO}$ oxidation on $\mathrm{Pd}(100)$ proceeding under near ambient pressure conditions. J. Phys. Chem. Lett. 2012, 3, 3182-3187. [CrossRef] [PubMed]

6. Frenken, J.; Groot, I. Live observations of catalysts using high-pressure scanning probe microscopy. In Operando Research in Heterogeneous Catalysis; Frenken, J., Groot, I., Eds.; Springer International Publishing: Cham, Switzerland, 2017; pp. 1-30.

7. Hendriksen, B.L.M.; Bobaru, S.C.; Frenken, J.W.M. Looking at heterogeneous catalysis at atmospheric pressure using tunnel vision. Top. Catal. 2005, 36, 43-54. [CrossRef]

8. Gao, F.; McClure, S.M.; Cai, Y.; Gath, K.K.; Wang, Y.; Chen, M.S.; Guo, Q.L.; Goodman, D.W. CO oxidation trends on Pt-group metals from ultrahigh vacuum to near atmospheric pressures: A combined in situ PM-IRAS and reaction kinetics study. Surf. Sci. 2009, 603, 65-70. [CrossRef]

9. Ryczkowski, J. IR spectroscopy in catalysis. Catal. Today 2001, 68, 263-381. [CrossRef]

10. Gustafson, J.; Shipilin, M.; Zhang, C.; Stierle, A.; Hejral, U.; Ruett, U.; Gutowski, O.; Carlsson, P.A.; Skoglundh, M.; Lundgren, E. High-energy surface X-ray diffraction for fast surface structure determination. Science 2014, 343, 758-761. [CrossRef] [PubMed] 
11. Stierle, A.; Gustafson, J.; Lundgren, E. Surface-sensitive X-ray diffraction across the pressure gap. In Operando Research in Heterogeneous Catalysis; Frenken, J., Groot, I., Eds.; Springer International Publishing: Cham, Switzerland, 2017; pp. 59-87.

12. Lundgren, E.; Zhang, C.; Merte, L.R.; Shipilin, M.; Blomberg, S.; Hejral, U.; Zhou, J.; Zetterberg, J.; Gustafson, J. Novel in situ techniques for studies of model catalysts. Acc. Chem. Res. 2017, 50, 2326-2333. [CrossRef]

13. Zhou, J.; Blomberg, S.; Gustafson, J.; Lundgren, E.; Zetterberg, J. Visualization of gas distribution in a model AP-XPS reactor by PLIF: CO oxidation over a Pd(100) catalyst. Catalysts 2017, 7, 29. [CrossRef]

14. Zellner, A.; Suntz, R.; Deutschmann, O. Two-dimensional spatial resolution of concentration profiles in catalytic reactors by planar laser-induced fluorescence: NO reduction over diesel oxidation catalysts. Angew. Chem. Int. Ed. 2015, 54, 2653-2655. [CrossRef] [PubMed]

15. Matera, S.; Reuter, K. First-principles approach to heat and mass transfer effects in model catalyst studies. Catal. Lett. 2009, 133, 156-159. [CrossRef]

16. Matera, S.; Reuter, K. When atomic-scale resolution is not enough: Spatial effects on in situ model catalyst studies. J. Catal. 2012, 295, 261-268. [CrossRef]

17. Zhou, J.; Blomberg, S.; Gustafson, J.; Lundgren, E.; Zetterberg, J. Simultaneous imaging of gas phase over and surface reflectance of a Pd(100) single crystal during CO oxidation. J. Phys. Chem. C 2017, 121, 23511-23519. [CrossRef]

18. Onderwaater, W.G.; Taranovskyy, A.; van Baarle, G.C.; Frenken, J.W.M.; Groot, I.M.N. In situ optical reflectance difference observations of CO oxidation over Pd(100). J. Phys. Chem. C 2017, 121, 11407-11415. [CrossRef] [PubMed]

19. Matera, S.; Reuter, K. Transport limitations and bistability for in situ CO oxidation at $\mathrm{RuO}_{2}(110)$ : First-principles based multiscale modeling. Phys. Rev. B 2010, 82. [CrossRef]

20. Roos, M.; Kielbassa, S.; Schirling, C.; Häring, T.; Bansmann, J.; Behm, R.J. Scanning mass spectrometer for quantitative reaction studies on catalytically active microstructures. Rev. Sci. Instrum. 2007, 78. [CrossRef] [PubMed]

21. Tan, C.K.C.; Delgass, W.N.; Baertsch, C.D. Spatially resolved in situ FTIR analysis of CO adsorption and reaction on $\mathrm{Pt} / \mathrm{SiO}_{2}$ in a silicon microreactor. Appl. Catal. B Environ. 2009, 93, 66-74. [CrossRef]

22. Zetterberg, J.; Blomberg, S.; Gustafson, J.; Evertsson, J.; Zhou, J.; Adams, E.C.; Carlsson, P.A.; Aldén, M.; Lundgren, E. Spatially and temporally resolved gas distributions around heterogeneous catalysts using infrared planar laser-induced fluorescence. Nat. Commun. 2015, 6, 7076. [CrossRef]

23. Blomberg, S.; Zhou, J.; Gustafson, J.; Zetterberg, J.; Lundgren, E. 2D and 3D imaging of the gas phase close to an operating model catalyst by planar laser induced fluorescence. J. Phys. Condens. Matter 2016, 28. [CrossRef]

24. Mantzaras, J. Progress in non-intrusive laser-based measurements of gas-phase thermoscalars and supporting modeling near catalytic interfaces. Prog. Energy Combust. Sci. 2019, 70, 169-211. [CrossRef]

25. Matera, S.; Maestri, M.; Cuoci, A.; Reuter, K. Predictive-quality surface reaction chemistry in real reactor models: Integrating first-principles kinetic monte carlo simulations into computational fluid dynamics. ACS Catal. 2014, 4, 4081-4092. [CrossRef]

26. Matera, S.; Blomberg, S.; Hoffmann, M.J.; Zetterberg, J.; Gustafson, J.; Lundgren, E.; Reuter, K. Evidence for the active phase of heterogeneous catalysts through in situ reaction product imaging and multiscale modeling. ACS Catal. 2015, 5, 4514-4518. [CrossRef]

27. Kee, R.J.; Coltrin, M.E.; Glarborg, P.; Zhu, H. Chemically Reacting Flow: Theory, Modeling, and Simulation; John Wiley \& Sons: Hoboken, NJ, USA, 2017.

28. Zanier, F.; Michelon, N.; Canu, P. Design and characterization of a stagnation flow reactor for heterogeneous microkinetic studies. Chem. Eng. J. 2017, 315, 67-82. [CrossRef]

29. Zetterberg, J.; Blomberg, S.; Zhou, J.; Gustafson, J.; Lundgren, E. Planar laser induced fluorescence applied to catalysis. In Operando Research in Heterogeneous Catalysis; Springer International Publishing: Cham, Switzerland, 2017; pp. 131-149.

30. Kohsehoinghaus, K. Laser techniques for the quantitative detection of reactive intermediates in combustion systems. Prog. Energy Combust. Sci. 1994, 20, 203-279. [CrossRef]

31. Aldén, M.; Bood, J.; Li, Z.; Richter, M. Visualization and understanding of combustion processes using spatially and temporally resolved laser diagnostic techniques. Proc. Combust. Inst. 2011, 33, 69-97. [CrossRef] 
32. Hanson, R.K. Planar laser-induced fluorescence imaging. J. Quant. Spectrosc. Radiat. Transf. 1988, 40, 343-362. [CrossRef]

33. Zhou, J.; Pfaff, S.; Lundgren, E.; Zetterberg, J. A convenient setup for laser-induced fluorescence imaging of both $\mathrm{CO}$ and $\mathrm{CO}_{2}$ during catalytic $\mathrm{CO}$ oxidation. Appl. Phys. B 2017, 123, 87. [CrossRef]

34. Gudmundson, F.; Persson, J.L.; Försth, M.; Behrendt, F.; Kasemo, B.; Rosen, A. OH gas phase chemistry outside a Pt catalyst. J. Catal. 1998, 179, 420-430. [CrossRef]

35. Fernandes, V.R.; Van den Bossche, M.; Knudsen, J.; Farstad, M.H.; Gustafson, J.; Venvik, H.J.; Grönbeck, H.; Borg, A. Reversed hysteresis during CO oxidation over $\operatorname{Pd}_{75} \mathrm{Ag}_{25}(100)$. ACS Catal. 2016, 6, 4154-4161. [CrossRef]

36. Rosell, J.; Bai, X.S.; Sjoholm, J.; Zhou, B.; Li, Z.; Wang, Z.; Pettersson, P.; Li, Z.; Richter, M.; Aldén, M. Multi-species PLIF study of the structures of turbulent premixed methane/air jet flames in the flamelet and thin-reaction zones regimes. Combust. Flame 2017, 182, 324-338. [CrossRef]

(C) 2019 by the authors. Licensee MDPI, Basel, Switzerland. This article is an open access article distributed under the terms and conditions of the Creative Commons Attribution (CC BY) license (http://creativecommons.org/licenses/by/4.0/). 